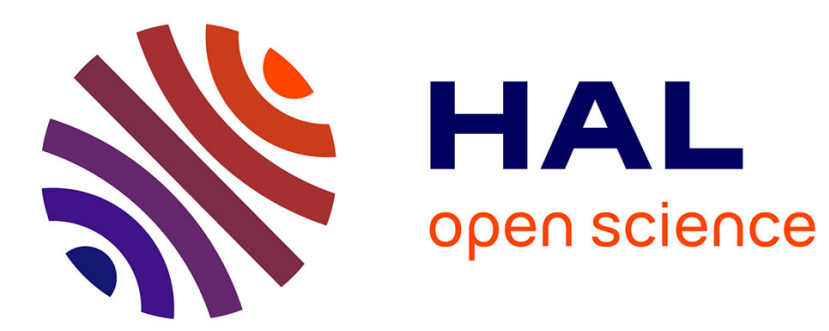

\title{
LUMINESCENCE DES ALLIAGES TERNAIRES GaxIn1-xP NON DOPÉS ET DOPÉS AU SOUFRE
}

\author{
Jacques Chevallier
}

\section{To cite this version:}

Jacques Chevallier. LUMINESCENCE DES ALLIAGES TERNAIRES GaxIn1-xP NON DOPÉS ET DOPÉS AU SOUFRE. Journal de Physique Colloques, 1974, 35 (C3), pp.C3-207-C3-213. 10.1051/jphyscol:1974330 . jpa-00215578

\section{HAL Id: jpa-00215578 https://hal.science/jpa-00215578}

Submitted on 1 Jan 1974

HAL is a multi-disciplinary open access archive for the deposit and dissemination of scientific research documents, whether they are published or not. The documents may come from teaching and research institutions in France or abroad, or from public or private research centers.
L'archive ouverte pluridisciplinaire HAL, est destinée au dépôt et à la diffusion de documents scientifiques de niveau recherche, publiés ou non, émanant des établissements d'enseignement et de recherche français ou étrangers, des laboratoires publics ou privés. 


\title{
LUMINESCENCE DES ALLIAGES TERNAIRES Ga $\mathbf{I n}_{1-x} \mathbf{P}$ NON DOPÉS ET DOPÉS AU SOUFRE
}

\author{
J. CHEVALLIER
}

Laboratoire de Physique des Solides, C.N.R.S., 1, place A.-Briand, 92190 Meudon-Bellevue, France

\begin{abstract}
Résumé. - Les spectres de photoluminescence et de cathodoluminescence des alliages $\mathrm{Ga}_{x} \operatorname{In}_{1-x} \mathrm{P}$ non intentionnellement dopés sont étudiés à basse température $(4,2 \mathrm{~K}$ et $20 \mathrm{~K})$ dans toute la gamme de compositions. Pour $0,69<x<1$, les spectres se composent d'excitons liés au carbone neutre et au soufre neutre. Ces raies fines sont accompagnées par une large transition à cinétique rapide attribuée à la présence d'azote résiduel. Pour $0,63<x<0,69$, outre cette large transition apparaît une raie haute énergie interprétée comme une transition donneur-bande de valence. Dans la gamme $x<0,63$, deux transitions dominent. A partir de mesures de photoluminescence à température variable, nous avons attribué ces deux transitions à des transitions donneur-bande de valence et donneur-accepteur.

Après dopage au soufre, les spectres des alliages de composition $x<0,63$ ne sont pas modifiés en ce qui concerne leur position énergétique. Pour $x>0,69$, seules les raies dues au soufre et à l'azote subsistent. Au voisinage de la composition $x=0,69$, les spectres sont composés de transitions caractéristiques des matériaux à bande interdite directe (transition niveau-bande et donneuraccepteur) et des matériaux à bande interdite indirecte (transitions excitoniques).

Un point de désaccord subsiste cependant entre d'une part le comportement du rapport des pics de luminescence en fonction du taux de dopage et de la composition et d'autre part l'écart énergétique $E_{\Gamma}-E_{\text {HE. }}$ Dans la première donnée, on s'attendrait à une énergie d'ionisation du donneur de l'ordre de quelques meV alors que dans la seconde, cette énergie serait de $35 \mathrm{meV}$ environ.
\end{abstract}

\begin{abstract}
The photoluminescence and cathodoluminescence spectra of non-intentionally doped $\mathrm{Ga}_{x} \operatorname{In}_{1-x} \mathbf{P}$ alloys are studied at low temperature $(4.2 \mathrm{~K}$ and $20 \mathrm{~K})$ in the whole range of compositions. For $0.69<x<1$, the spectra consist of narrow lines due to the recombination of excitons bound to neutral carbon and sulphur. Apart from these lines, we observe a large transition attributed to excitons bound to N-N pairs. This transition is characterized by a short decay time. For $0.63<x<0.69$, the large transition subsists and a donor-valence band transition appears at higher energy. In the range $x<0.63$, we see two peaks. From photoluminescence measurements at various temperature, we deduce the nature of these two peaks : a donor-valence band and a donor-acceptor pair transition.

After doping by sulphur, the peaks have the same energy position for alloys $x<0.63$. If $x>0.69$, the exciton lines due to sulphur and nitrogen are still present. Near the composition $x=0.69$, the spectra are composed of transitions characteristic of direct band gap alloys (free-bound and bound-bound transitions) and of indirect band gap alloys (excitonic transitions).

A disagreement exists about the value of the donor ionization energy. From the energy difference $E_{\Gamma}-E_{\mathrm{HE}}$, the ionization energy is found to be about $35 \mathrm{meV}$ whereas a value of a few meV only would explain the behaviour of the peak ratio versus doping level and composition.
\end{abstract}

1. Introduction. - Parmi les composés semiconducteurs à large bande interdite, les alliages $\mathrm{Ga}_{x} \mathrm{In}_{1-x} \mathrm{P}$ présentent théoriquement un intérêt certain du point de vue de l'électroluminescence. Si nous définissons par $x_{\mathrm{c}}$ la composition de l'alliage pour lequel les énergies des bandes interdites directe et indirecte sont égales, pour des compositions un peu inférieures à $x_{\mathrm{e}}$, les alliages présentent la particularité de posséder une bande interdite directe permettant une luminescence dans le spectre visible jusqu'au jaune inclus. On peut ainsi espérer de bien meilleures efficacités lumineuses de diodes qu'avec les autres composés ternaires comme $\mathrm{GaAs}_{1-x} \mathbf{P}_{x}$ et $\mathrm{Ga}_{x} \mathrm{Al}_{1-x}$ As [1]. Avant d'opti- miser les caractéristiques du matériau en vue de fabriquer des diodes, il est nécessaire de bien connaître les processus de recombinaison radiative des cristaux non intentionnellement dopés dans toute la gamme de compositions. A l'aide de techniques comme la photoluminescence à température fixe ou variable, la cathodoluminescence et les mesures d'effet Hall, nous avons cherché à déterminer l'origine des transitions de luminescence observées. Dans une seconde étape, nous avons étudié la luminescence des alliages dopés au soufre, cette impureté étant destinée à servir de dopant dans la partie $n$ des jonctions en raison de ses qualités déjà testées dans les diodes au phosphure de 
gallium [2]. Nous montrons quels changements apporte un tel dopage par rapport aux cristaux non dopés, en particulier au voisinage de la composition $x_{\mathbf{c}}$ du point de croisement des bandes.

2. Croissance des cristaux et techniques expérimentales. - En raison des hauts points de fusion des alliages $\mathrm{Ga}_{x} \mathrm{In}_{1-x} \mathrm{P}$ et de leur forte pression de décomposition à ces températures, la technique de croissance en solution est bien adaptée à la synthèse de ces cristaux. Elle doit permettre en outre une plus grande pureté du matériau. La méthode utilisée a déjà été décrite en détail [3], [4], [5]. Elle consiste à alimenter un bain d'indium de façon continue en gallium et en phosphore issus respectivement de $\mathrm{GaP}$ solide et de la vapeur. La pointe de l'ampoule où se produit la cristallisation est maintenue à température constante.

Après polissage, les cristaux sont analysés en microsonde de Castaing. Afin d'écarter l'hypothèse d'une erreur systématique sur nos valeurs de la composition $x$, des mesures parallèles ont été effectuées sur un même cristal, de composition voisine de $50 \%$, par notre laboratoire et par IBM (*). Nos valeurs sont systématiquement supérieures à celles de IBM de $\Delta x=0,01$.

Les quantités d'impuretés résiduelles et celles $\mathrm{du}$ soufre volontairement introduit $\left(2 \times 10^{17}\right.$ à $\left.2 \times 10^{18} \mathrm{~cm}^{-3}\right)$ ont été déterminées par spectrographie de masse à étincelle. Dans les cristaux non dopés, les impuretés dominantes sont le carbone et l'oxygène à des taux de l'ordre de 30 ppm (at) [6]. Les quantités de soufre et de silicium sont de l'ordre de $0,3 \mathrm{ppm}$ et celle d'azote de $1 \mathrm{ppm}$.

Pour les mesures de photoluminescence, les cristaux sont excités par les radiations émises par une lampe HBO $(100 \mathrm{~W}$ ou $200 \mathrm{~W})$ à vapeur de mercure. Un spectromètre muni d'un réseau à 1220 traits par millimètre analyse le spectre émis par le cristal. La résolution est de 1 à $2 \mathrm{meV}$. Pour les expériences à température constante, le cristal plonge directement dans le liquide cryogénique alors que dans l'appareillage à température variable, il est refroidi par le gaz d'hélium qui circule en permanence autour de lui. Dans ce cas, une chaîne de régulation assure une stabilité en température de l'ordre de $1 / 10^{\mathrm{e}}$ de degré.

Toutes les mesures de cathodoluminescence ont été réalisées au laboratoire Thomson-CSF de Corbeville (MM. Decremoux et Hirtz). Les cristaux sont bombardés par des électrons accélérés sous $20 \mathrm{kV}$. Les courants électroniques sont de l'ordre de $100 \mu \mathrm{A}$. Les impulsions de tension durent $1 \mu$ s environ et leur temps de descente est de 30 ns. Etant donné les surfaces excitées les densités de paires électron-trou créées sont situées dans la gamme $10^{23} \mathrm{~cm}^{-3} \cdot \mathrm{s}^{-1}$ (à faible injection) à $10^{25} \mathrm{~cm}^{-3} \cdot \mathrm{s}^{-1}$ (à forte injection). Les mesures sont possibles entre $6 \mathrm{~K}$ et $300 \mathrm{~K}$.

(*) Nous remercions vivement MM. Onton, W. Reuter et J. D. Kuptsis (IBM, Yorktown Heights, New York), et M. Rouy (CNRS; Bellevue) d'avoir permis que ces mesures soient menées à bien.
3. Luminescence des cristaux non intentionnellement dopés. - Afin de déterminer avec précision la structure de bande des alliages dans toute la gamme de compositions et à toute température, des mesures d'électroréflectance et de piézoréflectance ont été menées par MM. Alibert et Bordure au CEES Montpellier. Ces travaux ont été publiés récemment [7], [8]. La composition $x_{\mathrm{c}}$ à laquelle les énergies des bandes $\Gamma$ et $\mathrm{X}$ sont égales est 0,69 à très basse température. A cette composition $E_{\mathrm{X}}=E_{\Gamma}=2,32 \mathrm{eV}$

3.1 Alliages de Composition $x>0,69$. - Dans la gamme de composition $0,69 \leqslant x \leqslant 1$, les alliages possèdent une bande interdite indirecte. La figure 1 présente un spectre typique de la composition $x=0,80$. Il se compose d'une large transition à basse énergie et de raies fines à haute énergie dont l'intensité est très sensible aux variations de température. On remarque que l'une d'entre elles $(2,295 \mathrm{eV})$ diminue moins rapidement que les autres quand la température augmente. En raison de leur finesse et de leur position énergétique voisine de la bande interdite, nous attribuons ces cinq transitions à des excitons liés à des impuretés neutres. La transition dont l'énergie est la plus élevée ne peut impliquer des donneurs neutres, excepté le silicium. L'extrapolation à $x=1$ conduit en effet à une énergie supérieure à $2,310 \mathrm{eV}$, énergie maximum des photons émis lors de la recombinaison d'un exciton lié à un donneur comme le tellure, le soufre ou le sélénium [9]. Le silicium conduit à des transitions d'énergie voisine de 2,315 eV dans GaP [10] alors que les accepteurs $(\mathrm{Cd}, \mathrm{Zn}, \mathrm{Mg}$ et $\mathrm{C})$ donnent des énergies de transition sans phonon comprises entre $2,319 \mathrm{eV}$ et $2,324 \mathrm{eV}$ [11], [12]. Nous pensons que la raie excitonique à haute énergie $(2,308 \mathrm{eV})$ est due au carbone en raison des quantités importantes détectées par spectro-

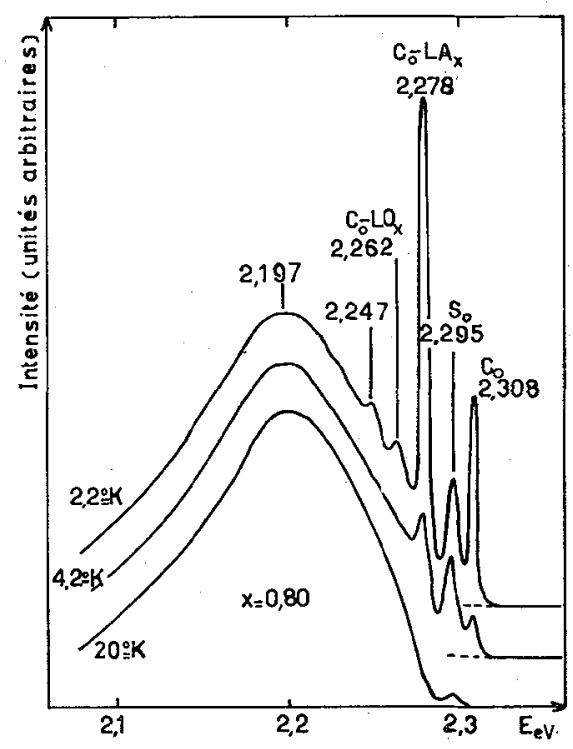

Fig. 1. - Spectre de photoluminescence d'un alliage $\mathrm{Ga}_{x} \operatorname{In}_{1-x} \mathrm{P}$ non intentionnellement dopé à bande interdite indirecte. 
graphie de masse. D'autre part, les matériaux non intentionnellement dopés, à bande interdite indirecte sont toujours de type $\mathrm{p}$. L'extrapolation à $x=1$ donne $2,318 \mathrm{eV}$, soit $6 \mathrm{meV}$ en dessous de l'énergie de la recombinaison de l'exciton lié au carbone neutre. Un tel écart entre la valeur extrapolée et la valeur réelle dans $\mathrm{GaP}$ a déjà été signalé par Onton dans le cas de l'exciton lié au tellure neutre [13]. Les transitions à $2,278 \mathrm{eV}$ et $2,262 \mathrm{eV}$ sont attribuées à des répliques phononiques de la transition précédente mettant en jeu respectivement des phonons $\mathrm{LA}_{\mathrm{X}}$ et $\mathrm{LO}_{\mathrm{X}}$. Les énergies des phonons sont en accord avec celles déterminées à partir de l'énergie des phonons dans GaP [9] et dans InP [14] en supposant qu'elles obéissent à une loi linéaire avec la composition. Le pic d'énergie 2,295 eV est probablement dû à la recombinaison d'un exciton lié à un donneur neutre. Le soufre est le donneur le plus vraisemblable, sa concentration étant nettement supérieure à celle $\mathrm{du}$ tellure et du sélénium. On remarque encore un écart énergétique de $6 \mathrm{meV}$ entre la valeur extrapolée et la valeur réelle dans GaP.

Ces raies fines sont accompagnées d'une transition très large. La largeur à mi-hauteur est typiquement de $120 \mathrm{meV}$. Une transition de paires donneuraccepteur est peu probable pour deux raisons. Dans $\mathrm{GaP}$, la transition de paires $\mathrm{C}-\mathrm{S}$, avec des teneurs comparables à celles de nos cristaux, a une largeur à mi-hauteur environ 4 fois plus faible. D'autre part, une étude en régime transitoire en cathodoluminescenice a montré que le temps de déclin de cette transition est inférieur à 30 ns. Ce temps est beaucoup trop faible pour une transition de paires dans un matériau à bande interdite indirecte. Macksey et al. ont trouvé des transitions tout à fait similaires dans les alliages $\mathrm{Ga}_{x} \mathrm{In}_{1-x} \mathrm{P}$ [15], [16] et $\mathrm{GaAs}_{1-x} \mathrm{P}_{x}$ [17] dopés à l'azote. Ils attribuent cette luminescence à la recombinaison d'excitons liés à des paires $\mathrm{N}-\mathrm{N}$. De tels spectres sont observés dans la gamme des

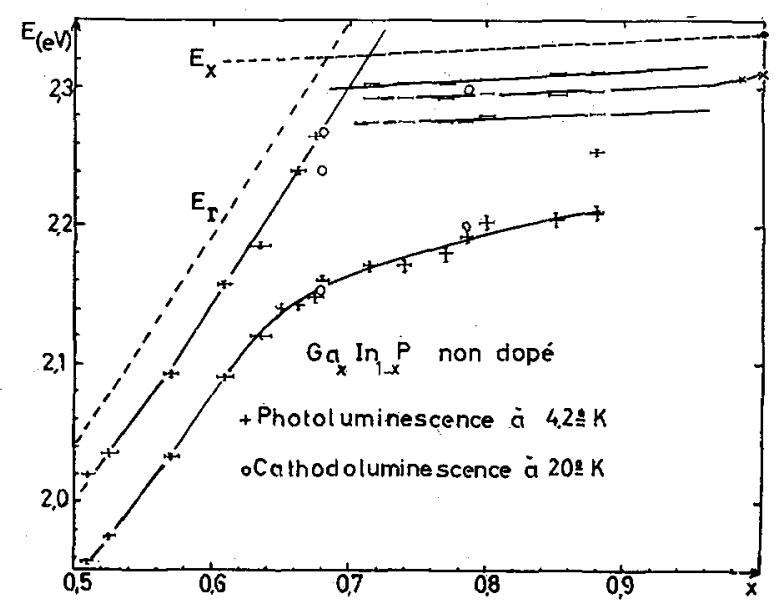

FIG. 2. - Structure de bande et transitions de luminescence des alliages $\mathrm{Ga}_{x} \operatorname{In}_{1-x} \mathrm{P}$ non dopés. Les énergies des pics de photoluminescence $(x)$ et de la bande interdite indirecte de $\mathrm{GaP}(\cdot)$ sont tirées des références [18] et [19]. compositions supérieures à 0,70 , aussi bien en photoluminescence qu'en cathodoluminescence comme le montre la figure 2 .

3.2 Alliages de composition $x<0,69$. - Dans le domaine des compositions $0,63<x<0,69$, les cristaux non intentionnellement dopés sont de type $\mathrm{p}$. On retrouve la même transition très large que dans le cas des alliages à bande interdite indirecte et nous lui attribuerons la même origine. Il apparaît aussi une transition à plus haute énergie. Pour $x<0,63$, les cristaux sont de type n. Leur spectre de photoluminescence est présenté sur la figure 3 . Il se compose de deux pics d'énergie inférieure à $E_{\mathbf{g}}$. La raie basse énergie a une largeur à mi-hauteur de l'ordre de $40^{\circ} \mathrm{meV}$ : elle est donc de nature différente de celle très large que l'on trouve pour $x>0,63$. Etant donné l'énergie $E_{\mathrm{g}}$ de la bande interdite, nous attribuons ces pics à des transitions niveau-bande et donneuraccepteur. Nous développerons plus loini la détermination de la nature du niveau mis en jeu dans la transition haute énergie. Récemment, un pic d'énergie supérieure à celle des deux précédents a été mis en évidence sur certains cristaux [6], [20]. Aux incertitudes près, son énergie coïncide avec celle de la bande interdite $\Gamma$, à $4,2 \mathrm{~K}$, déterminée par électroréflectance et piézoréflectance.

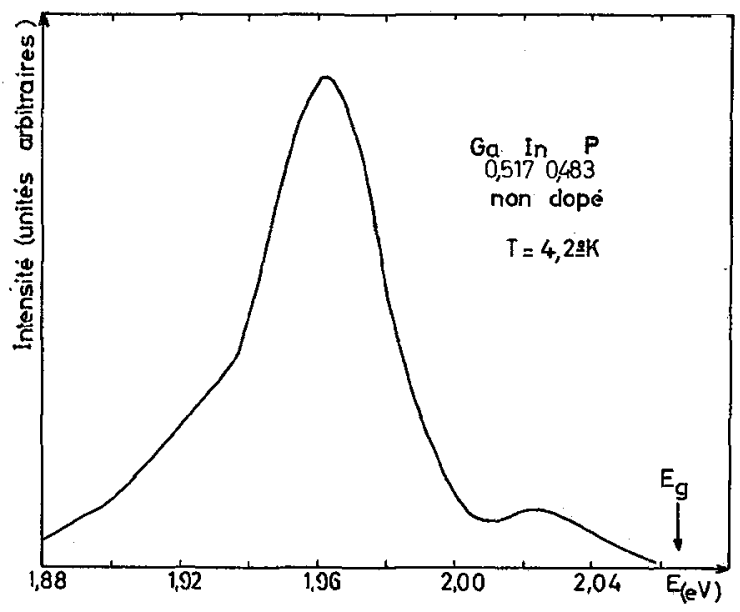

FrG. 3. - Photoluminescence d'un alliage non intentionnellement dopé à bande interdite directe. La flèche indique l'énergie de la bande interdite déduite des mesures d'électroréflectance et de piézoréflectance à $10 \mathrm{~K}$ environ.

4. Luminescence des cristaux dopés au soufre. Les alliages à bande interdite indirecte présentent des spectres identiques à ceux des matériaux non dopés excepté du côté haute énergie où seul l'exciton lié au soufre neutre est visible.

Pour les compositions riches en InP, les spectres sont encore composés de deux pics et, comme le montre la figure 4, la transition basse énergie suit la variation de la bande interdite jusqu'à $x=0,695$. Par cathodoluminescence sous forte injection, nous 


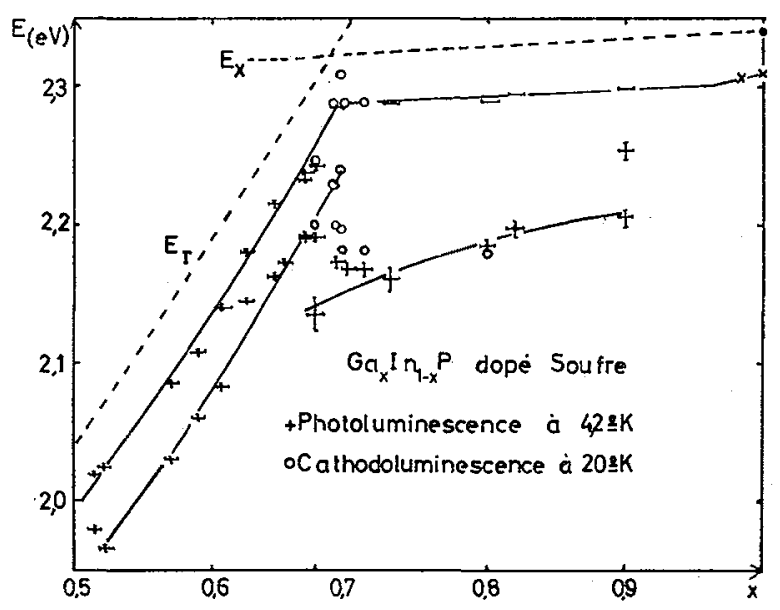

FIG. 4. - Transitions de luminescence des alliages $\mathrm{Ga}_{x} \operatorname{In}_{1-x} \mathrm{P}$ dopés au soufre. Même remarque que pour la figure 2 .

avons enregistré les spectres à $20 \mathrm{~K}$ en deux points d'un même cristal, ces deux points ayant des compositions fort peu différentes de 0,69 (Fig. 5). La même densité d'excitation a été utilisée. Lorsque la composition augmente légèrement, le pic basse énergie et le pic le plus intense ne se déplacent pas en énergie alors que le pic intermédiaire passe de $2,230 \mathrm{eV}$ à $2,240 \mathrm{eV}$. En raison de son énergie, ce pic est attribué à la transition donneur-accepteur : il suit la variation de la bande de conduction $\Gamma$. Le pic le plus intense est dû, d'après ce qui précède, à l'exciton lié au soufre neutre alors que le pic basse énergie est dû à la présence d'excitons liés à des paires N-N. Ces deux transitions sont liées à la bande de conduction $X$. On voit donc apparaître dans ce domaine de composition des recombinaisons caractéristiques à la fois des matériaux à bande interdite directe et des matériaux à bande interdite indirecte. Des effets similaires ont été observés sur $\mathrm{GaAs}_{1-x} \mathrm{P}_{x}$ au voisinage du point de croisement [21]. Sur le cristal le plus riche en GaP,

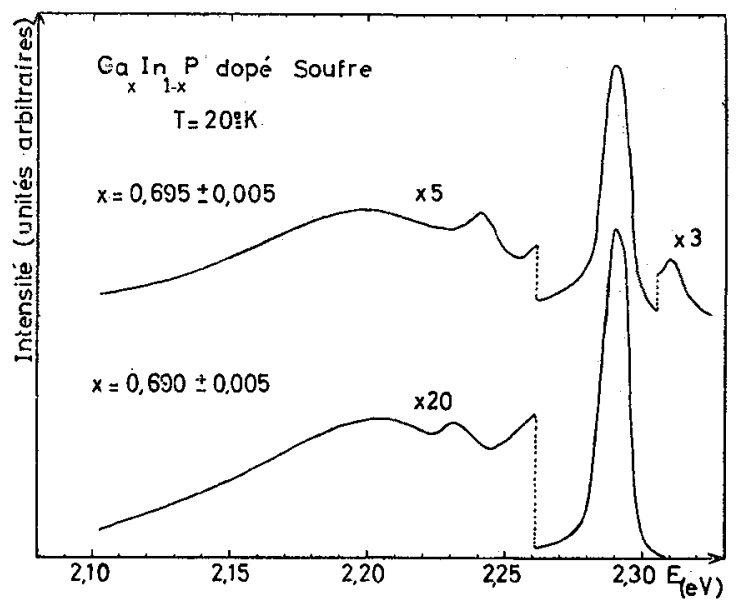

FrG. 5. - Photoluminescence d'un alliage dopé au soufre de composition proche de celle du point de croisement des bandes à $20 \mathrm{~K}$. on voit aussi apparaître un pic haute énergie dont la largeur à mi-hauteur est de 4,6 meV. Ce pic est sans doute d'origine excitonique. Notons enfin que la disparition de la transition niveau-bande à $x>0,695$ s'explique par le fait que pour de telles compositions le niveau donneur (cas d'une transition donneurbande de valence) ou la bande de conduction $\Gamma$ (cas d'une transition bande de conduction-accepteur) ont une énergie supérieure à la bande de conduction $X$ et sont par conséquent vides d'électrons.

5. Etude des transitions dans les alliages à bande interdite directe dopés ou non. - 5.1 RÉsultats EXPÉRIMENTAUX. - L'évolution des intensités intégrées de chacune des deux transitions en fonction de la température est reportée sur la figure 6 dans le cas d'un cristal non dopé. Le pic basse énergie décroît très vite à partir de $36 \mathrm{~K}$, température à laquelle le pic haute énergie augmente. Après passage par un maximum, le pic haute énergie est prépondérant. Des comportements tout à fait analogues sont obtenus. avec les matériaux dopés au soufre.

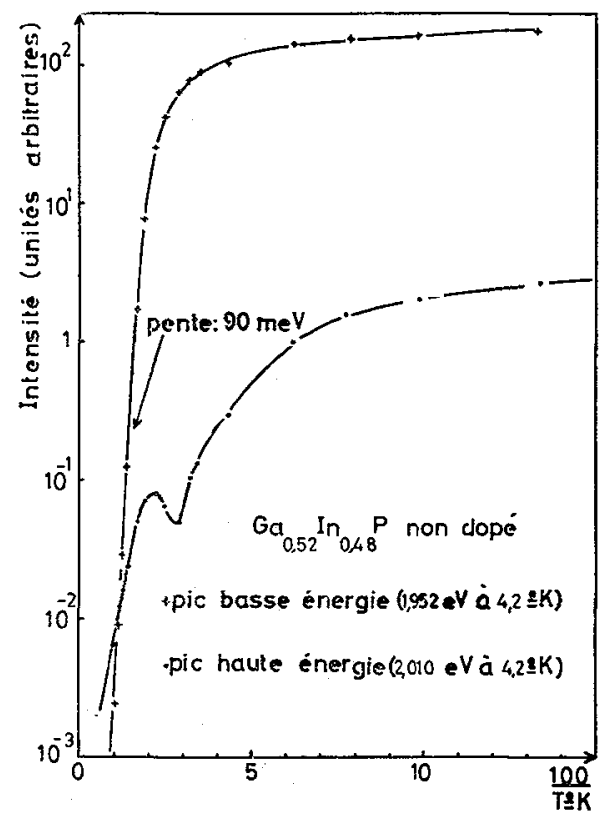

FIG. 6. - Variation des intensités des raies haute et basse énergie en fonction de la température sur un alliage $\mathrm{Ga}_{0,52} \operatorname{In}_{0,48} \mathrm{P}$ non dopé.

5.2 INTERPRÉTATION. - D'après les mesures électriques [22], le nombre de porteurs $n_{\mathrm{H}}$ déduit de la constante de Hall varie très peu avec la température. Le niveau de Fermi reste toujours «bloqué " près de la baride de conduction. Il en résulte que le donneur profond ( $\sim 35$ à $40 \mathrm{meV}$ ) est neutre quelle que soit la température ; il ne peut s'ioniser à $36 \mathrm{~K}$. La diminution de l'intensité de la transition basse énergie est donc due à l'ionisation de l'accepteur. Nous interprétons l'accroissement de la transition haute énergie, 
à $36 \mathrm{~K}$, par une augmentation du nombre de porteurs minoritaires (les trous) dans la bande de valence au moment où commence l'ionisation des accepteurs. La transition haute énergie impliquant un porteur minoritaire est donc une transition donneur-bande de valence.

La pente déduite de la variation de l'intensité du pic basse énergie avec la température est de $90 \mathrm{meV}$. Cette valeur élevée n'est pas l'énergie d'ionisation de l'accepteur. Considérons en effet le schéma des transitions représenté sur la figure 7. Dishman [23] a

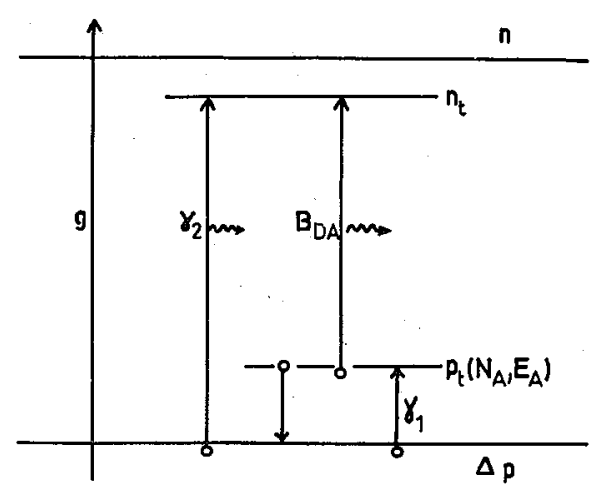

FIG. 7. - Schéma des transitions électroniques dans le cas d'un alliage à bande interdite directe (type n).

montré que le taux de recombinaison radiative d'une transition de paires pouvait s'approcher par la forme simple $B_{\mathrm{DA}} n_{t} p_{t}$ où $B_{\mathrm{DA}}$ est une constante indépendante de la distance entre le donneur et l'accepteur et indépendante de la température, $n_{t}$ et $p_{t}$ étant les concentrations respectives de donneurs neutres et d'accepteurs neutres. Ecrivons le bilan des trous sur le niveau accepteur, en régime stationnaire :

$$
\begin{array}{rl}
\frac{\mathrm{d} p_{t}}{\mathrm{~d} t}=-B_{\mathrm{DA}} n_{t} p_{t}+\gamma_{1} & p\left(N_{\mathrm{A}}-p_{t}\right)- \\
& \quad-\gamma_{1} N_{\mathrm{v}} \beta p_{t} \exp -\frac{E_{\mathrm{A}}}{k T}=0
\end{array}
$$

où $\gamma_{1}$ est le coefficient de capture des trous par les accepteurs ionisés,

$N_{\mathrm{v}}$ est la densité d'états de la bande de valence,

$\beta$ est la dégénérescence du niveau accepteur,

$E_{\mathrm{A}}$ est l'énergie d'ionisation de l'accepteur.

Le taux de recombinaison radiative de la transition de paires est :

$$
I_{\mathrm{BE}}=B_{\mathrm{DA}} n_{t} p_{t}
$$

Celui de la transition niveau-bande est :

$$
I_{\mathrm{HE}}=\gamma_{2} n_{t} p
$$

où $\gamma_{2}$ est le coefficient de capture des trous par les donneurs neutres.
On déduit que :

$$
\frac{I_{\mathrm{HE}}}{I_{\mathrm{BE}}}=\frac{\gamma_{2} p}{B_{\mathrm{DA}} p_{t}}
$$

De 1'éq. (1), on tire :

$$
\frac{p}{p_{t}}=\frac{B_{\mathrm{DA}} n_{t}+\gamma_{1} N_{\mathrm{v}} \beta \exp -\frac{E_{\mathrm{A}}}{k T}}{\gamma_{1}\left(N_{\mathrm{A}}-p_{t}\right)} .
$$

Le cristal de type n. En se plaçant dans le régime des faibles excitations, on a :

$$
p \simeq \Delta p, \quad n_{t} \simeq n_{t_{0}}, \quad N_{\mathrm{A}}-p_{t} \simeq N_{\mathrm{A}} .
$$

On a donc :

$$
\frac{I_{\mathrm{HE}}}{I_{\mathrm{BE}}}=\frac{\gamma_{2} n_{t_{0}}}{\gamma_{1} N_{\mathrm{A}}}+\frac{\gamma_{2} \beta N_{\mathrm{v}}}{B_{\mathrm{DA}} N_{\mathrm{A}}} \exp -\frac{E_{\mathrm{A}}}{k T} .
$$

A très basse température, le second terme est faible par rapport au premier. Le rapport des intensités varie peu : ceci est beaucoup plus net pour les matériaux dopés au soufre de même composition. Quand la température augmente, le deuxième terme devient prépondérant. De la partie linéaire de la variation du rapport $I_{\mathrm{HE}} / I_{\mathrm{BE}}$ avec la température, on déduit l'énergie d'ionisation de l'accepteur (Fig. 8). Compte tenu de la variation de $N_{\mathrm{v}}$ avec la température, on trouve une énergie $E_{\mathrm{A}}$ égale à $54 \mathrm{meV}$. Cette valeur est en accord avec l'écart énergétique entre les deux pics $(58 \mathrm{meV})$. La pente de $90 \mathrm{meV}$ trouvée pour le pic basse énergie sur la figure 6 ne représente donc pas l'énergie d'ionisation de l'accepteur. La raison en est la diminution du nombre $\Delta p$ de porteurs minori-

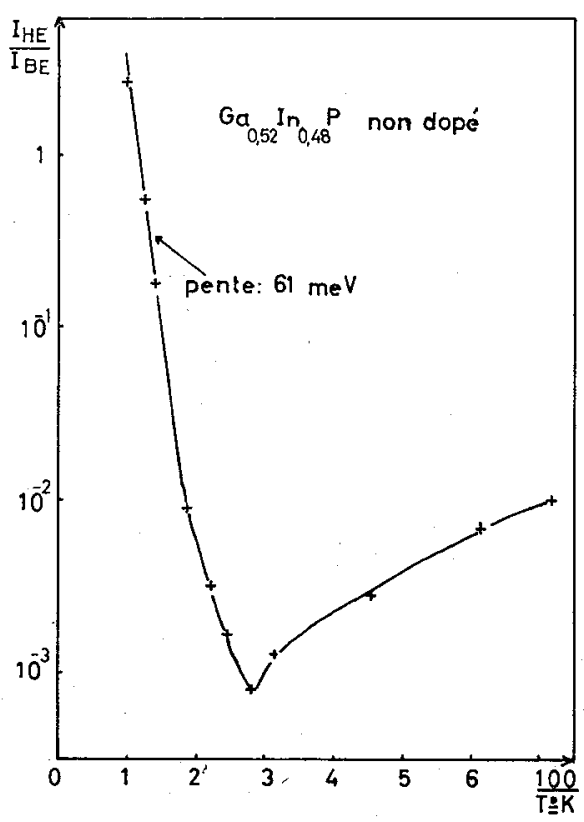

Fig. 8. - Variation du rapport des intensités des pics haute énergie (HE) et basse énergie (BE) en fonction de la température pour un alliage $\mathrm{Ga}_{0,52} \operatorname{In}_{0,48} \mathrm{P}$ non dopé. La pente est de $61 \mathrm{meV}$. En tenant compte de la variation de la densité d'état $N_{\mathrm{v}}$, l'énergie d'ionisation $E_{\mathrm{A}}$ est de $54 \mathrm{meV}$. 
taires créés en excès au-delà de $46 \mathrm{~K}$, dont l'origine se situe peut-être dans la diminution de la durée de vie non radiative lorsque la température augmente.

5.3 INFLUENCE DU DOPAGE SUR LE RAPPORT DES INTENSTTÉS DES PICS HAUTE ET BASSE ÉNERGIE. - Dans l'analyse qui précède, l'énergie d'ionisation du donneur n'est pas intervenue. En particulier, un tel raisonnement est applicable si le niveau donneur est immergé dans la bande de conduction. Par des mesures électriques, nous avons montré que le soufre introduisait un niveau hydrogénoïde dans les alliages à bande interdite strictement directe $(x<0,54)$. A basse température, l'expression du rapport $I_{\mathrm{HE}} / I_{\mathrm{BE}}$ se réduit à :

$$
\frac{I_{\mathrm{HE}}}{I_{\mathrm{BE}}}=\frac{\gamma_{2} n_{t 0}}{\gamma_{1} N_{\mathrm{A}}} .
$$

La variation de $I_{\mathrm{HE}} / I_{\mathrm{BE}}$, à une composition donnée $(x=0,52)$ et à une température donnée $(T=25 \mathrm{~K})$, provient donc de la variation de $n_{t_{0}}$ (Fig. 9). Les mesures électriques ont montré par ailleurs que le niveau de Fermi à l'équilibre est situé très près de la bande de conduction quelle que soit la température. Ceci entraîne que le niveau donneur impliqué dans les transitions de luminescence est superficiel ou est immergé dans la bande de conduction, car sinon $n_{t_{0}}$ serait constant.

La figure 9 présente aussi les variations du rapport $I_{\mathrm{HE}} / I_{\mathrm{BE}}$ avec la composition dans la gamme $0,52<x<0,67$. Les mesures ont été effectuées sur plusieurs tranches d'un même lingot de façon à assurer un taux de dopage constant. On observe une diminution $\mathrm{du}$ rapport lorsque la composition augmente. D'autre part, les mesures électriques ont montré, à une température donnée, que la concen-

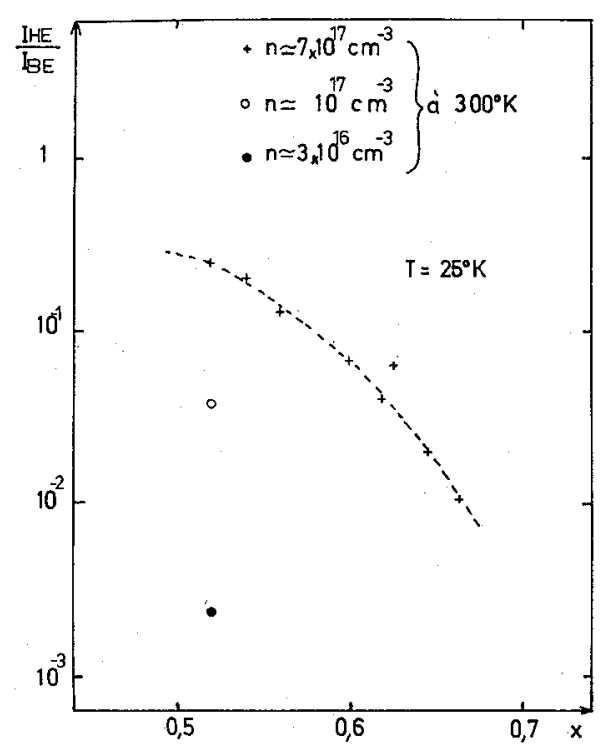

Fıo. 9. - Variation du rapport des intensités des pics haute et basse énergie en fonction du taux de dopage et de la composition à̀ $25 \mathrm{~K}$. tration de porteurs diminue lorsque $x$ augmente [22]. En adoptant une valeur de $35 \mathrm{meV}\left(E_{\Gamma}-E_{\mathrm{HE}}\right)$ pour l'énergie d'ionisation du donneur impliqué en luminescence, on constate aisément à partir de la position du niveau de Fermi que $n_{t_{0}}$ ne varie pas. L'évolution observée s'explique par contre si le donneur a une énergie d'ionisation beaucoup plus faible.

6. Discussion. - Des considérations qualitatives qui précèdent, il résulte un désaccord entre les valeurs de l'énergie d'ionisation du donneur déduite de $E_{\Gamma}-E_{\mathrm{HE}}$ et celle déduite du comportement de $I_{\mathrm{HE}} / I_{\mathrm{BE}}$ avec le taux de dopage et la composition. Ceci provient en partie de l'incertitude sur $E_{T}-E_{\mathrm{HE}}$ qui est dérivé de mesures indépendantes de photoluminescence, d'électroréflexion et de piézoréflexion.

Nous avons montré que l'hypothèse d'une transition donneur-bande de valence et d'une transition donneur-accepteur explique bien le comportement de l'intensité de luminescence en fonction de la température pour les alliages à bande interdite directe de type $\mathrm{n}$ sans préjuger de l'énergie d'ionisation du donneur. L'énergie de la bande interdite déterminée par les techniques de modulation est supérieure ou égale à l'énergie de ces transitions.

Macksey [16] obtient des spectres analogues aux nôtres dans la gamme $0,5<x<1$. S'appuyant sur les résultats de cathodoluminescence de Onton [24], il interprète les pics haute et basse énergie à $x \simeq 0,60$ comme étant respectivement dus à un état résonnant du piège azote dans la bande de conduction et à une transition bande à bande. La théorie [15] prévoit par contre que l'écart énergétique entre les deux pics ne cesse d'augmenter lorsque la teneur en GaP diminue alors que, expérimentalement, cet écart reste constant, en particulier pour $x<0,50$ [6]. Enfin, Macksey n'observe pas l'accroissement résonnant attendu pour la raie haute énergie à la composition $x=0,71$ pour laquelle $E_{\mathrm{N}} \simeq E_{\Gamma}$. Ceci est normal étant donné l'estimation différente de la bande interdite. A partir des nouvelles valeurs de la bande interdite directe, on trouve que les bandes se croisent à $x_{\mathrm{c}}=0,69$ et que l'effet d'accroissement résonnant est attendu à $x=0,65$.

7. Conclusions. - Les cristaux non intentionnellement dopés, à bande interdite directe, possèdent des transitions de luminescence résultant des impuretés résiduelles comme le carbone, le soufre et l'azote. La transition excitonique de paires $\mathrm{N}-\mathrm{N}$ subsiste au voisinage du point de croisement alors qu'apparait une transition donneur-bande de valence. Une transition donneur-accepteur domine les spectres à basse température pour $x<0,60$. Ce modèle est en accord avec une théorie simple faisant intervenir le bilan détaillé sur le niveau accepteur. Par contre, nous n'avons pu expliquer le comportement du rapport des intensités avec le taux de dopage et la composition 
à partir des valeurs de la bande interdite déterminée par les techniques de modulation.

Après dopage au soufre, les alliages à bande interdite strictement directe ou indirecte ne sont pas modifiés excepté la prédominance dans ces derniers de l'exciton lié au soufre neutre. Pour quelques compo- sitions voisines du point de croisement $(x \simeq 0,69)$, on a pu mettre en évidence des transitions caractéristiques à la fois des alliages à bande interdite directe (transition niveau-bande et donneur-accepteur) et de ceux à bande interdite indirecte (transitions excitoniques).

\section{Bibliographie}

[1] Archer, R. J., Electrochem. Soc. Meeting, Los Angeles, California, Spring 1970, paper 66.

[2] Logan, R. A., White, H. G. et Wiegmann, W., Appl. Phys. Lett. 13 (1968) 139.

[3] Rodot, H., Hruby, A, et Schnemer, M., J. Crystal Growth 3 (1968) 305.

[4] Chevallier, J. et Rodot, H., C. R. Hebd. Séan. Acad. Sci. B 271 (1970) 1037.

[5] Laugier, A. et Chevallier, J., Phys. Stat. Sol. (a) 7 (1971) 427.

[6] Chevallier, J. et Laugier, A., Phys. Stat. Sol. (a) 8 (1971) 437.

[7] Alibert, C., Bordure, G., Laugier, A. et Chevallier, J,, Phys. Rev. B 4 (1972) 1301.

[8] Laugier, A., Alibert, C. et Chevallier, J., J. Physique 35 (1974) C3-77.

[9] Dean, P. J., Phys. Rev. 157 (1967) 655.

[10] Vink, A. T., Bosman, A. J., Van der does de Bye, J. A. W. et Peters, R. C., J. of Luminescence 5 (1972) 57-68.

[11] VINK, A. T.' et Peters, R. C., J. of Luminescence 3 (1970) 209-229.

[12] Dean, P. J., Faulkner, R. A. et Kimura, S., Solid. State Commun. 8 (1970) 929.
[13] Onton, A. et Chicotka, R. J., Phys. Rev. B 4 (1971) 1847

[14] Semiconductors and Semimetals, 3 WiLlardson et BeER, p. 87.

[15] Scifres, D. R., Macksey, H. M., Holonyak, N., Jr., Dupuis, R. D., ZaCk, G. W., Duke, C. B., Kleiman, G. C. et Kunz, A. B., Phys. Rev. B 5 (1972) 2206.

[16] Macksey, H. M., Holonyak, N. Jr., Dupuis, R. D., Camprell, J. C. et Zack, G. W., J. Appl. Phys. 44 (1973) 1333.

[17] Holonyak, N., Jr., Dupuis, R. D., Macksey, H. M., Craford, M. G. et Groves, W. O., J. Appl. Phys. 43 (1972) 4148.

[18] Logan, R. A., Dean, P. J., White, H. G. et Wiegmann, W., J. Appl. Phys. 42 (1971) 2328.

[19] Dean, P. J. et Thomas, D. G., Phys. Rev. 150 (1966) 690.

[20] Laugier, A. et Chevaluier, J., Solid State Commun. 10 (1972) 353.

[21] Alferov, Zh. I., Garbuzov, D. Z., Zhilyaev, Yu. V. et Kop'ev, P. S., Sov. Phys. Semicond. 6 (1972) 589.

[22] Chevallier, J., Phys. Stat. Sol. (a) 14 (1972) 531.

[23] Dishman, J. M., Phys. Rev. B 3 (1971) 2588.

[24] Onton, A. et Lorenz, M. R., Proceedings of the 1970 Symposium on GaAs and related compounds, Aachen, Germany, 1970, 222 (non publié).

\section{DISCUSSION}

P. J. DEAN - Do your data contain any evidence concerning the recent suggestion by workers at STL that the lowest conduction band minima in $\mathrm{Ga}_{x} \mathrm{In}_{1-x} \mathrm{P}$ are from the symmetry point $\mathrm{L}$ near $x \sim 0.7$ ?

J. Chevallier. - Non. Dans la variation des spectres à basse température en fonction de l'énergie nous n'avons observé qu'une seule " cassure » située à $x \simeq 0,70$.

H. KRESSEL. - We have recently published the results of a study in $\operatorname{In}_{0.5} \mathrm{Ga}_{0.5} \mathrm{P}$ doped with $\mathrm{Te}$ indicating a donor ionization energy of $7 \mathrm{meV}$ (KRESSel H., Nuese C. J. and Ladany, I. J. Appl. Phys., July, 1973). How does this value compare to that of sulfur ?

J. Chevallier. - Dans les alliages $\mathrm{Ga}_{0,5} \operatorname{In}_{0,5} \mathrm{P}$, l'énergie d'ionisation du soufre est tout à fait similaire à celle que vous indiquez pour le tellure. Le soufre se comporte comme un centre hydrogénoïde (CHEvallier, J., Phys. Stat. Sol. (a), 14 (1972), 531). 